\title{
Construccion de paz desde la memoria colectiva y la educación
}

\author{
Holman G. Zamora Muñoz* \\ William Ricardo Zambrano Ayala*
}

\section{Resumen}

El contexto de posconflicto producto del acuerdo de paz entre el gobierno colombiano y la guerrilla de las Fuerzas Armadas Revolucionarias de Colombia-Ejército del Pueblo (FARC-EP), abre la oportunidad de generar una nueva construcción social y colectiva alejada de discursos y narrativas violentas. En razón con lo anterior, se propone realizar un análisis de construcción de paz desde la memoria colectiva y la educación. Para tal fin, se sigue una investigación teórica, cualitativa de carácter evaluativo, argumentativo, analítico y prospectivo sobre el objetivo trazado.

Se concluye que la educación es fundamental en los procesos de reflexión de todos los actores académicos, que deben promover escenarios de participación para la reconstrucción de la memoria y la búsqueda de la verdad. Cruzar los relatos sociales, entre ellos los grupos al margen de la ley, es el trabajo sobre memoria que constituye el tejido social y la convivencia en las comunidades víctimas del conflicto.

Palabras clave: educación, memoria colectiva, participación, paz, posconflicto

\section{Abstract}

The post-conflict context resulting from the peace agreement between the Colombian government and the Revolutionary Armed Forces of Colombia-People's Army (FARC-ep) guerrilla opens the opportunity to generate a new social and collective construction away from violent discourses and narratives. So, it is proposed to carry out an analysis of peace-building from the collective memory and education. To this end, a theoretical, qualitative research of an evaluative, argumentative, analytical and prospective nature is carried out on the objective set.

It concludes that education is fundamental in the processes of reflection of all academic actors, who must promote participation scenarios for the reconstruction of memory and the search for truth. Crossing social narratives, including groups outside the law, is the work on memory that constitutes the social fabric and coexistence in the communities that are victims of the conflict.

Keywords: collective memory, education, involvement, peace, postconflict

\footnotetext{
* Doctorado Periodismo. Análisis del discurso en la prensa colombiana. Universidad Complutense de Madrid. Maestría. Comunicación Social y Periodismo. Institución Universitaria Politécnico Grancolombiano. Líder Nacional de Investigación Escuela de Comunicación y Bellas Artes de la Corporación Unificada Nacional de Educación Superior (cun). Contacto: holman_zamora@cun.edu.co

** Posdoctorado en Dispositivos Digitales, Doctor en la Sociedad de la Información y del Conocimiento. Magíster en Comunicación Social. Especialista en Televisión de la Universidad Javeriana. Especialista en Gerencia de Recursos Humanos. Docente en la Corporación Unificada Nacional de Educación Superior (cun). Contacto: william_zambrano@cun.edu.co
} 


\section{Introducción}

La memoria colectiva en un país como Colombia tiene una validez inmensa y más si se realiza en temas coyunturales como el posconflicto, en el que las víctimas han sabido labrar un camino de silencio, temor, padecimientos y vicisitudes. En realidad, en el desarrollo continuo de la memoria colectiva no hay, como en la historia, líneas de separación claramente trazadas, sino solamente límites irregulares e inciertos. El presente (entendido como extendiéndose sobre una cierta duración, la que interesa a la sociedad de hoy) no se opone al pasado del mismo modo en que se distinguen dos periodos históricos vecinos, uno, el pasado ya no existe pues se vive de los recuerdos, y el otro, el presente que parte del perdón hasta donde alcanza la memoria. Los dos periodos tienen la misma intensidad en la realidad.

En este contexto han estado presentes diferentes actores, entre ellos: una sociedad que ha sufrido los hechos sangrientos propiciados por este grupo insurgente; la educación que mediante sus actores académicos debe interpretar y hacer una lectura clara y coherente de la realidad del proceso, con el fin de proponer relatos que contribuyan a una memoria colectiva de los problemas coyunturales del país y de la sociedad en este proceso de construcción de paz. Surge así el siguiente interrogante: ¿qué análisis se puede hacer sobre la construcción de paz desde la memoria colectiva y la educación?

En consecuencia, se plantean los siguientes objetivos: analizar la construcción de paz desde la memoria colectiva y la educación. Realizar un estado del arte de la memoria colectiva sobre el proceso del conflicto. Desarrollar un marco teórico que incluya una mirada crítica desde diversos autores. Dicha reflexión, interpretación y análisis será un aporte a la comunidad académica, al país y a la sociedad en temas de paz y del posconflicto en su transición hacia las condiciones de justicia social, convivencia, verdad, tolerancia y perdón.

\section{Propuesta metodológica}

Se realiza una investigación teórica, cualitativa de carácter evaluativo, argumentativo, analítico y prospectivo, que según Hernández, FernándezCollado y Baptista (2014) implica un conjunto de procesos de recolección y vinculación de datos cualitativos para responder al planteamiento del problema y a los objetivos propuestos. En lo cualitativo se tienen en cuenta los cuatro momentos propuestos por Denzin y Lincoln (2015): el primero corresponde al hermenéutico comprensivo (Londoño y Castañeda, 2010); el segundo, descriptivo; el tercero, argumentativo (Hernández, Fernández-Collado y Baptista, 2013) y el cuarto, analítico.
Para el estado del arte, se realizó una revisión bibliográfica que inició con la búsqueda documental en bases de datos, libros, tesis de grado (maestría y doctorado), artículos científicos publicados en revistas indexadas, informes y documentos oficiales. Posteriormente, los documentos encontrados se ordenaron según las categorías de análisis mencionados en la presente investigación, se organizó cada investigación consultada aplicando la siguiente matriz: contextualización, objetivo, metodología y conclusiones. La revisión documental representó una rigurosidad que requería la búsqueda, clasificación e interpretación de la 
información para ubicar los antecedentes del objeto de estudio.

Para el abordaje del marco teórico, se elaboraron documentos de apoyo que incluyeron una mirada de los conceptos desde diversos autores y dialogar con ellos a partir de un enfoque crítico (reflexionar sobre cuáles concepciones nos distanciamos y cuáles incluimos en la investigación) y por último, se realizó una aproximación teórica construida desde nuestro papel de investigadores. Para llevar a cabo este método, se requirió de una lectura rigurosa, la

\section{Antecedentes de análisis y teóricos}

El primer acuerdo fue el que dio término a la Guerra de los Mil Días, que fue refrendado con el asesinato del general Rafael Uribe Uribe, en un complot que comprometió al Vaticano, la Iglesia católica y los terratenientes conservadores y liberales. Lo mismo sucedió con Guadalupe Salcedo luego de los supuestos acuerdos de paz con las guerrillas liberales del llano que surgieron del crimen de Jorge Eliecer Gaitán.

Los pactos de paz del gobierno Betancur se convirtieron en un baño de sangre a partir de 1984, porque los industriales y terratenientes que manejan las fuerzas militares terminaron firmando los acuerdos de paz entre 1989 y 1991, lo que propició una alianza entre el Estado, los narcotraficantes y las fuerzas militares en el proceso del primer acuerdo durante la era del expresidente Belisario Betancur.

Algunos autores han realizados investigaciones sobre conflicto, entre ellos, Ricoeur (2000) que estudió la representación de la memoria como un ejercicio de evocación del pasado y representación de lo ausente; Halbwachs (2004) analizó realización de fichas, y el análisis e interpretación de los documentos. Para lo anterior, se utilizó la observación participante que, según Guber, "es el medio ideal para realizar descubrimientos, para examinar críticamente los conceptos teóricos y anclarlos en realidades concretas, poniendo en comunicación distintas reflexividades" (2004, pp. 83-97). Sobre este proceso se destaca la afirmación hecha por los autores Ramírez, Gouveia, y Lozada (2011) que resaltan que esta técnica no se basa en lo que las personas dicen, hacen o piensan sino en el análisis que de ello hace el investigador.

los marcos institucionales de la memoria en los que se ubica la escuela y los niveles interrelacionados de memoria individual y colectiva.

Todorov (1995) investigó los abusos de la memoria y la posibilidad de trabajar la memoria ejemplarizante con base en experiencias dolorosas por las que ha atravesado nuestra sociedad; y Jelin (2001 y 2002) identificó la importancia del tema de las políticas de la memoria oficial, que tienen como principal escenario de lucha y tensión la escuela. En los últimos años se han realizado investigaciones sobre la memoria como reconstrucción histórica y, a la vez, como mecanismo de catarsis. También, existen estudios acerca de construcción de la memoria en Sudáfrica durante el post Apartheid (Castillejo, 2006), en Chile posterior a la era Pinochet (Guichard y Henríquez, 2011) y en Argentina después de la dictadura. Estas investigaciones buscan reconstruir una narrativa paralela a la oficial que resignifique la experiencia y los relatos de las víctimas como mecanismos de afianzamiento del tejido social y la inclusión de este en los relatos oficiales. 
Antequera (2011) reconstruyó la historia desde las comunidades como resultado de la segunda guerra mundial, que generó la discusión en torno al nivel de la objetividad que tenía el relato de los vencedores. Dado que "el uso de la comunicación moderna para la reafirmación de valores y posiciones de poder, implicó un documentado cambio en la significación de los sufrimientos" (Mate, 2008, p. 43). Esto implicó la manera en que se observaba a las minorías y significó que la socialización de los relatos de la memoria sería importante para aprender de la experiencia y no repetir los hechos. Estas prácticas bien pueden expresarse en lo que se denominan los "vehículos de la memoria" (Uprimny y Saffon, 2009, p. 19). En tanto los libros, los archivos, las conmemoraciones, la oralidad, etc., permiten el resguardo y la socialización de la memoria.

Jelin (2002) en la segunda mitad de la década del noventa, lideró un proyecto con jóvenes investigadores sobre las memorias de la represión del Cono Sur, en momentos en que los regímenes militares impusieron su bota, entre los años sesenta y ochenta, en países como Argentina, Chile, Brasil y Paraguay. El programa apuntó a la creación de una red de intelectuales públicos de la región, preocupados por el estudio de la memoria societal y demás temas relacionados con ella.

Dussel, Finocchio y Gojman (2003) trazaron como objetivo que la consigna de Nunca Más se convierta en un acuerdo básico de la sociedad argentina, moviéndose en una lógica de memoria ejemplarizante. La intención de su investigación fue trabajar el tema de la memoria del horror en los contextos escolares, desarrollando desde un inicio una conceptualización básica en torno a los temas de Estado y sociedad y su relación con la democracia y el autoritarismo, abordando los diferentes enfoques de la dictadura militar, vivida entre 1976 y 1983. La "memoria del horror" es una memoria situada, desde este periodo hasta inicios del siglo XXI, mediante la cual se desarrolla un balance de la memoria colectiva, las formas y los lugares en los que se sostiene y la relación de los acontecimientos, posteriores a 1983, con los derechos humanos.

En el contexto colombiano, Zuluaga y Marín (2006) desarrollan un estudio sobre la memoria activa del saber pedagógico desde la perspectiva de memoria colectiva silenciada. Esta categoría tiene que ver con el reconocimiento de la memoria individual que los distintos sujetos tienen de las prácticas educativas y formativas de las cuales han hecho parte como maestros, estudiantes, ciudadanos o miembros de una institución social. Para las autoras, un saber pedagógico, que se constituye en recuerdo y expresión de memoria, es activo y duradero en la medida en que se reflexione sobre él.

Sánchez y Rodríguez (2009) investigaron la memoria en un país en guerra, concluyeron que hasta terminado el año 2009 no se habían difundido trabajos de tipo pedagógico que pudieran involucrar explícitamente la historia reciente de Colombia del conflicto interno. Para estas historiadoras no se encuentra en la agenda educativa una política de incorporación de la memoria del conflicto armado colombiano, ni como tema curricular ni como objeto de trabajo pedagógico. La discusión tiene como trasfondo el tema de las políticas de la memoria, que se constituyen a su vez en una categoría de análisis.

En esta misma línea, Silva et al. (2010) indagaron la memoria del periodo de la Violencia en Colombia,que es vivido a mediados del siglo xx. El tema es trabajado en seis instituciones educativas, evidenciando la situación de esta temática en el marco del debate actual entre la 
memoria oficial y otras memorias. Las ciencias sociales, su enseñanza y la relación que puede establecer con la memoria están compuestas por tensiones y tendencias inmersas en las lógicas de saber y poder. La memoria surge, así como un saber emergente, logrando una expansión en el campo académico y político, expresada en distintas manifestaciones ligadas fundamentalmente a los sucesos conflictivos que han caracterizado el país.

Desde la perspectiva de la subjetivación del sujeto, en la que se establecen unas condiciones de posibilidad para que este se vea, se narre, se exprese, se juzgue y se domine, se encuentran una serie de iniciativas de trabajo que han decantado, por medio de su relación con la memoria, la categoría de subjetivación política en la que se ubican a Herrera y Ramírez (2009), Gómez (2009) y Rodríguez (2009).

Las anteriores investigaciones dan cuenta de manera implícita de la preocupación por el carácter subjetivo en torno al tema del sujeto y su abordaje en la construcción del conflicto a través de la oralidad, la narrativa y su relación con la experiencia vivida. La categoría de memoria colectiva de manera explícita se logra ubicar en una serie de iniciativas del 2005 a la fecha, de manera inicial en el Colectivo de historia oral, conformado en su mayoría por profesores e investigadores que dejan las bases de una memoria activa desde el saber pedagógico, mediante la cual se pueden documentar los relatos de las víctimas que hasta el momento han sido silenciadas y sometidas a procesos de invisibilización.

Es así que la memoria de una sociedad se extiende hasta donde ella puede, es decir, hasta donde alcanza la memoria de los grupos de que está compuesta. No es por mala voluntad, antipatía, repulsión o indiferencia por lo que olvida tal cantidad de acontecimientos y personajes antiguos. Es porque los grupos que conservaban su recuerdo han desaparecido. Halbwachs y Díaz complementan "si la duración de la vida humana se doblara o triplicara, el campo de la memoria colectiva, medido en unidades de tiempo, sería mucho más extenso" (1995, p. 2159).

Algunos autores que han trabajado la resolución de conflictos centran su atención en tres aspectos fundamentales de la práctica pedagógica en una iniciativa curricular de paz: el entrenamiento y práctica de formas dialógicas que incentiven la participación, el debate y la discusión, en las que prime el peso del argumento y no la posición de los hablantes; el manejo de emociones, de técnicas y métodos de resolución de conflictos que les permitan a las personas no solo poder resolver sus propios conflictos de forma creativa y crítica, sino colaborar además como mediadores en la resolución de muchos de los conflictos cotidianos que presenciamos y frente a los cuales por incompetencia o indiferencia tomamos la decisión de no actuar.

La memoria colectiva acompañada de las nuevas tecnologías se ha transformado, constituyéndose en un nuevo vínculo y soporte de la memoria contemporánea, siendo inherente al hombre conservar su memoria de acuerdo a ciertas posibilidades técnicas de registro y representación: la pintura, la escritura y las nuevas tecnologías. Esta iniciativa establece una relación directa entre la historia, la memoria, las tradiciones y las fuentes orales.

Para que se pueda hablar de memoria hace falta quelas partes delconflicto sobreelque seextiende estén en alguna medida bien diferenciadas. Cada uno de esos actores tiene una historia, por 
consiguiente se distinguen relatos, recuerdos y hechos. Pero lo que nos llama la atención es que en la memoria las similitudes pasan a un primer plano. En el momento en que considera su pasado, el grupo siente claramente que ha seguido siendo el mismo y toma conciencia de su identidad a través del tiempo. La construcción de memoria permite comprender de una manera significativa, entre otros temas, el conflicto social y político por el cual han atravesado los colombianos. Precisamente es aquí donde se valora la memoria como parte de las relaciones de significado de las víctimas, como una construcción simbólica y subjetiva, y como un ejercicio de evocación del pasado y representación de lo ausente (Ricoeur, 2000).

\section{Discusión y análisis}

En cualquier país del mundo la inversión en educación es la clave para pensar el progreso, el desarrollo y la justicia social, partiendo de entender, en el mismo orden, que gran parte de los problemas sociales vinculados a la pobreza, se encuentran relacionados con la distribución de la ignorancia, el acceso preferencial a la educación y una educación sistemáticamente diferente según la clase social a la que se pertenezca.

De forma directa, los altos niveles de violencia, delincuencia, drogadicción, desempleo, etc. que aquejan a muchos países del tercer mundo se encuentran articulados a individuos y grupos sociales con posibilidades escasas o limitadas de acceso a la educación, redundando esto en posibilidades escasas de inserción en el sistema productivo lo que incrementa el conflicto social.

De forma general la educación ha demostrado ser la estrategia fundamental para prevenir e intervenir el conflicto social en el largo plazo, al permitir a las personas analizar sus propios problemas con mayor claridad de cara al futuro. De este modo, una educación formal con calidad para todos, debe responder al reto de formar ciudadanos íntegros para el ejercicio de la participación, la democracia y la convivencia. La educación para la paz es un proceso de participación en el cual debe desarrollarse la capacidad crítica, esencial para los nuevos ciudadanos del mundo. Se deben enseñar y aprender soluciones a los conflictos, a la guerra, a la violencia, al terrorismo, a la explotación de género, a combatir el daño ambiental y oponerse a todo lo que sea contrario a la vida y a la dignidad humana. "Hay que aprender a comportarse para favorecer la transición de una cultura de guerra y de fuerza a una cultura de paz" (Mayor Zaragoza, 2003).

No obstante, los presupuestos y gastos públicos destinados para la inversión en educación se encuentran siempre en la balanza entre lo urgente y lo importante, entre los problemas a corto plazo y las necesidades a largo plazo del país. En este sentido, procesos de paz como los del Salvador, Nicaragua y otros, han mostrado la importancia de pensar el papel trascendental de la educación en el posconflicto para la reconstrucción, reintegración y reinserción social, más allá de resultados políticos o económicos para solventar coyunturas y crisis inmediatas.

Por eso, la educación para la paz es mucho más que un curso, es más que una materia de valores. Debe ser el contenido transversal de la educación. Pero este contenido no vendrá por sí solo ni tendrá un impacto real de cambio sino a través de la voluntad política, decidida y 
expresa, de los gobiernos, de los parlamentos y de los consejos municipales.

En este contexto surge la necesidad y el desafío pedagógico, no solo de promover en general el rol del conocimiento y la educación como soporte angular del posconflicto, sino además de proponer y diseñar una educación para la paz como fundamento pragmático que permita tanto a educadores como estudiantes hacer frente a las diversas formas que toma el conflicto social, esto implica pensar una serie de competencias axiológicas o formación en valores para el manejo del conflicto y la resolución asertiva y pacífica de problemas y conflictos.

Las nuevas sociedades, más por el influjo de la globalización y las tecnologías de la comunicación y la información, tienden a formas cada vez más diversas, plurales y cosmopolitas de vida, potenciando con ello mayor cantidad de encuentros con la diferencia y alteridad de individuos y culturas, lo que supone formar ciudadanos con unas altas competencias en la tolerancia, el diálogo y el comportamiento ético.

En Colombia es pertinente tener en cuenta que aunque existe una relación directa entre el conflicto político armado que podríamos entender como macro estructural y la violencia común o micro estructural o cotidiana, esta última cobra entre el $80 \%$ al $90 \%$ de las víctimas, lo que indica la necesidad de pensar desde el aula un currículo, no solo que contenga de manera transversal contenidos, metodologías y estrategias diseñadas para la reflexión crítica de esta situación, sino además que le permita a los educados tener elementos y capacidades para soportar, enfrentar y transformar dicha realidad.

Esta reflexión permite además hacer precisiones pragmáticas entorno al conflicto social y el conflicto armado de orden político mismo en el país, dado que los orígenes y permanencia de este último responden de forma directa a la alta desigualdad social, a la diferencia material de vida y posibilidades entre los que tienen mucho y los que se tienen solo a sí mismos, es decir, a la distancia o brecha social que genera la violencia estructural de la que emana tanto el conflicto social como el político a su vez. Sin embargo, el conflicto social se encuentra ligado a los altos niveles de frustración, desesperanza y resentimiento que conlleva la distancia social y la pobreza.

En este sentido, debe entenderse de forma clara que la firma de los acuerdos de la Habana y todas las dificultades posteriores a dichos acuerdos desde su refrendación a implementación, en el mejor de los casos, pone fin a un conflicto armado político entre uno de los grupos revolucionarios (FARC-EP) y el Estado.

Sin embargo, el conflicto social seguirá mientras continúen las formas de desigualdad, distancia y brecha social. Esto indica, que una cosa es hablar de pos conflicto, que podría enunciar (o suponer de forma ambigua o ingenua) la superación de esta distancia o brecha social y otra de pos acuerdo en referencia a lo pactado y sus posteriores dificultades de implementación.

Sin restarle importancia a esta precisión, de cara a la educación y sus objetivos en la formación de ciudadanos para la vida, más allá de los acuerdos y su coyuntura, implica pensar en cómo responder transversal y longitudinalmente a esa problemática cotidiana, a esa presión y estrés social responsable de más del $80 \%$ de los crímenes en el país que muestran de manera alarmante el asentamiento de una cultura violenta de resolución de conflictos que pasa fácilmente de la negación de la otredad a la eliminación del contrario. 
El acceso a la educación en diferentes circunstancias supone para muchos niños $y$ jóvenes el único lugar de protección, refugio y prevención ante las amenazas que encuentran incluso más allá de la calle, en sus propios hogares y familias, convirtiendo a la escuela en refugio material y físico de muchos tipos de agresiones a los que se encuentran expuestos los educandos. Por tanto, la educación y la escuela deben ofrecer protección material, cognitiva y psicológica, además de poder garantizar las condiciones de seguridad y bienestar físico y emocional de todos sus miembros.

La escuela debe restablecer y fortalecer los vínculos sociales y esto empieza por brindar a sus estudiantes una identidad como aprendices que les posibilite a su vez encontrar sentido a sus vidas y alejarlos de otros caminos e influencias que pueden hallar en los diferentes contextos como las armas, la drogadicción o la delincuencia. Este sentido de identidad y pertenencia debe además encontrarse encaminado a desarrollar actitudes, habilidades y competencias que fortalezcan sus capacidades analíticas y de juicio crítico para poder resistir y encaminar sus propios deseos y satisfacciones dentro de vías legales y ciudadanas.

El reto de cualquier proceso de paz o pos conflicto es invertir en educación como vía a la reinserción económica y social, radica en lograr que los desmovilizados y combatientes hallen opciones de vida donde encuentren futuro, fuera de la delincuencia y las armas.

Desde el propio Banco Mundial a autores como Stiglitz, Sen y muchos otros académicos del campo de la economía, recomiendan para la reconstrucción de cualquier país el papel central de la educación y el conocimiento como motores de desarrollo, crecimiento económico y bienestar social. La principal teoría se basa en el significado de la creación de un sólido sistema educativo como parte de la recuperación posconflicto (Infante, 2013).

La educación y el conocimiento son los principales mecanismos de desarrollo humano y movilidad social por tanto su importancia trasciende sin restarle valor, el crecimiento económico ligado a ella y la convierte en un valor en sí misma. Sin embargo, la educación en cualquier proceso de paz y fundamentalmente posterior a cualquier acuerdo, debe comprenderse no solo como un instrumento importante de desarrollo de las comunidades, sino que además implica un cambio de pensamiento y reconciliación de todos los actores sociales implicados en la guerra, sobre todo de aquellos que de alguna $u$ otra manera consideran que la guerra trae o les ha traído beneficios.

Dentro de este contexto, surge la necesidad de una educación para la paz, que neutralice y contrarreste una cultura del conflicto que ha legitimado la violencia como forma de resolver los problemas y en la cual se han instalado otra serie de antivalores sociales conexos, unidos por ejemplo al problema del narcotráfico, la cultura del vivo y la corrupción.

La educación para la paz, continuando una larga tradición y discusión filosófica, asume el conflicto como inherente a la convivencia e interacción social, como un proceso consustancial a la experiencia y a la existencia humana que conlleva al entendimiento de la paz no como negación o ausencia del conflicto, sino a comprender el conflicto como la necesidad de búsqueda de alternativas para mejorar la convivencia por tanto como motor de cambios hacia formas más justas de vivir juntos.

Cualquier proceso de enseñanza aprendizaje, tanto individual como colectivo debe partir 
de un principio de educabilidad que supone la posibilidad de que los seres, sobre todo los humanos, tenemos la capacidad de concebirnos como seres en continua evolución y cambio, en permanente construcción y por tanto inacabados y susceptibles de ser educados por otros y en capacidad de educar a otros.

Desde el punto de vista de una pedagogía para la paz, en el marco del pos acuerdo, esta educabilidad parte del supuesto de una cultura de la violencia y la guerra que ha negado la

\section{Conclusiones}

Lo principal de esta reflexión es que primero hay que terminar el conflicto para luego abrir una fase de construcción de la paz estable y duradera. Eso fue lo que se convino con las FARC-EP en el Acuerdo General que se firmó en agosto de 2012. Si entendemos la construcción de la paz como un ejercicio para reforzar normas y hacer valer derechos, tenemos un marco para ponderar mejor los intereses de la justicia con los de la paz. Siempre y cuando entendamos justicia en el sentido amplio de la palabra, como un sistema equitativo de cooperación con sus correspondientes derechos y deberes, que todos aceptan y que regulan su conducta.

En tal sentido, se debe iniciar un diálogo en todos los sectores para discutir entre todos cómo desarrollar y poner en práctica lo que se ha acordado en La Habana. Entre estos sectores se encuentra la educación. Lo principal es entender la paz como una oportunidad, como una gran palanca de cambio para hacer lo que no hemos logrado hacer en sesenta años de guerra.

La desintegración del tejido social y la degradación de valores éticos y morales que la guerra y el conflicto han logrado a través de la diferencia y cuya mayor dificultad radica en el manejo adecuado y asertivo de los conflictos y problemas, negándonos la oportunidad de reconocernos y aprender de la otredad.

Aunque no hay y no debe de haber un consenso sobre cómo educar a las generaciones por venir, la pluralidad y diversidad cada vez más evidente en nuestras sociedades implica configurar una serie de competencias y pautas de comportamiento ético y moral que logren una comprensión más humana y empática entre todos.

historia en la sociedad, reclaman a la educación lograr convertirse en lugar de restablecimiento y recomposición de derechos y valores sociales.

La educación debe responder al reto de formar ciudadanos capaces y competentes de participar, proponer y transformar críticamente esta realidad, pero lo que esto supone pedagógicamente pasa por comprender la escuela y por ende la educación como un escenario de encuentro y reconciliación para intervenir las marcas y huellas que ha dejado el conflicto social y la guerra, tanto a nivel psicológico como emocional en los individuos como en las comunidades o grupos.

De esta manera, el papel de la educación, el conocimiento y el acceso a la información, son tanto instrumentos para combatir la pobreza, como las formas de exclusión y marginación, así como de disminuir la probabilidad de la ocurrencia de la violencia de armada, política a común. El fomento del conocimiento, la información y el pensamiento crítico, fundamentos de la educación y de la academia, tienen sus detractores y enemigos en la guerra que, a diferencia de los efectos devastadores 
para la gran mayoría de la población, traen beneficios para algunos grupos y personas que a su sombra ven incrementado su poder y renta. De políticos a empresarios pasando por militares piensan la guerra como una industria de grandes divisas.

La participación política busca romper el vínculo entre política y armas y restablecer una regla básica de la sociedad, que nadie recurra a las armas para promover sus ideas políticas; y que nadie que esté en desacuerdo sea víctima de la violencia.

Es importante comprender que uno de los escenarios más golpeados y en muchas circunstancias blanco estratégico de la guerra y el conflicto mismo ha sido la escuela y la educación a través de tácticas sistemáticas que van desde la poca inversión en recursos para su financiación y funcionamiento institucional, la deslegitimación de sus actores y actividades, a la amenaza, persecución y desaparición de sus actores.

De esta forma la educación para la paz se propone como eje transversal a todos los currículos, entendiendo esta transversalidad, no solo como la aplicación de ciertos contenidos como formar en valores en determinadas asignaturas, o a diferentes áreas o momentos de aprendizaje, sino a una educación que permita poner en contacto y armonizar diferentes aspectos $\mathrm{y}$ dimensiones del individuo y lo social, pasando por lo medioambiental.

La educación tiene un compromiso y una responsabilidad en el cuidado de sí, de cada individuo y miembro de la comunidad; en el cuidado del otro, de todas las formas de otredad y alteridad; y de lo otro, en relación y soporte con todo lo que nos rodea y por tanto su reto es construir conocimiento y memoria de los individuos y de sus hechos, realidades y conflictos en general.

Así, el tema de la memoria se convierte en el contexto colombiano en una demanda política que no ha sido incorporada en el ámbito social, la cual reclama una serie de relatos y legado del conflicto a partir de las historias contadas por las víctimas en los procesos de construcción de paz, lo cual permite fortalecer el tejido social para promover el dialogo y la reconciliación.

Ahora bien, como afirma Marín "varios son los aprendizajes y las lecciones que nos dejan otros procesos de recuperación del pasado y tramitación de la verdad y la justicia en América Latina" (2010, p. 40). En primer lugar, los esfuerzos de países como Argentina, Brasil, Chile, El Salvador, Guatemala y Uruguay por enfrentarse con sus pasados y conectarlos con los procesos de transición democrática reflejan la importancia de las condiciones y los acontecimientos históricos de cada país en estos esfuerzos.

Lo anterior nos obliga a reconocer que los informes de memoria donde está consignada la verdad histórica, el ejercicio de levantamiento y condensación de los testimonios de las víctimas, los procesos de justicia con los victimarios y los ejercicios de reparación para las víctimas pueden ser en cualquier momento cuestionados desde distintos sectores. Es necesario valorar estos cuestionamientos, es incluso necesario que ocurran, ya que hacen parte de la actualización y pluralización a las que deben estar sometidas las memorias, las cuales no están para clausurar el pasado, sino para someterlo constantemente a escrutinio y discusión pública.

El proceso de paz necesariamente debe satisfacer los derechos de las víctimas, comenzando por la medida más efectiva de protección y no 
repetición: poner fin al conflicto. Igualmente, garantizar por igual los derechos constitucionales de los colombianos en todo el territorio. Asimismo, buscar transformar las condiciones del campo y reversar los efectos de la violencia. En consecuencia, se plantean las siguientes preguntas: ¿cómo cerrar la brecha entre lo urbano y lo rural, que ha sido el escenario del conflicto? ¿Qué planes y programas se pueden llevar a cabo para la construcción de paz que le dé un giro positivo a las condiciones de vida de la población?

\section{Referencias}

Antequera, J. (2011). Memoria histórica como relato emblemático. Consideraciones en medio de la emergencia de políticas de memoria en Colombia (tesis de maestría). Pontificia Universidad Javeriana, Bogotá. Recuperado de https:/ / bit.ly/2UHgjWo

Castillejo, A. (2006). Una laguna fundamental del sistema democrático. El Consejo Estatal de Medios Audiovisuales de España. Cuadernos de Comunicación e Innovación, 68, 61-71.

Denzin, N. y Lincoln, Y. (2015). Métodos de recolección y análisis de datos. Barcelona: Gedisa Editorial.

Dussel, I., Finocchio, S. y Gojman, S. (2003). Haciendo memoria en el país de nunca más. Buenos Aires: Eudeba.

Gómez, J. (2009). Voces que quieren hacerse oír. Narrativas de la memoria y el olvido en la escuela. En A. Jiménez y F. Guerra (comps.), Las luchas por la memoria (pp. 145-156). Bogotá: Universidad Distrital Francisco José de Caldas; Centro de Memoria, Paz y Reconciliación Distrital.

Guber, R. (2004). El salvaje metropolitano. Buenos Aires: Paidós.

Guichard, E. y Henríquez, G. (2011). Memoria histórica en Chile: una perspectiva intergeneracional desde Concepción. Revista Española de Investigaciones Sociológicas, 135(1), 3-25. Recuperado de https:/ / bit.ly/2HhzWwd

Halbwachs, M. (2004). Memoria colectiva. Zaragoza: Prensa Universitaria.

Halbwachs, M. y Díaz, A. (1995). Memoria colectiva y memoria histórica. Reis, 69, 209-219.

Hernández, R., Fernández-Collado, C. y Baptista, P. (2013). Metodología de la investigación. México D.F.: McGraw Hill Education.

Hernández, R., Fernández-Collado, C. y Baptista, P. (2014). Metodología de la investigación. México D.F.: McGraw Hill Education. 
Herrera, M. y Ramírez , L. (2009). Políticas de la memoria como forma de socialización y de subjetivación política: un análisis histórico sobre el tiempo presente. En A. Jiménez y F. Guerra (comps.), Las luchas por la memoria (pp. 21-62). Bogotá: Universidad Distrital Francisco José de Caldas; Centro de Memoria, Paz y Reconciliación Distrital.

Infante, A. (2013). El papel de la educación en situaciones de posconflicto: estrategias y recomendaciones. Hallazgos, 11(21), 223-245.

Jelin, E. (2001). Los niveles de la memoria: reconstrucciones del pasado dictatorial argentino. Entrepasados, 10(20), 9-34. Recuperado de https://bit.ly/37kZ8wN

Jelin, E. (2002). Los trabajo de la memoria. Buenos Aires: Siglo XXI.

Londoño, D. y Castañeda, L. (2010). La comprensión como método en las ciencias sociales. Revista Virtual Universidad Católica del Norte, 31, 227-252. Recuperado de https:/ / bit.ly/2SpXANI

Marín, J. (2010). La reconstrucción de la memoria histórica del conflicto colombiano en el actual proceso de Justicia y Paz. Alcances, desafíos y preguntas. Desafíos, 22(2), 31-70. Recuperado de https:/ / bit.ly/2SpmxIW

Mate, R. (2008). La herencia del olvido. Madrid: Errata naturae.

Mayor, F. (2003). Educación para la paz. Educación XX1, 6, 17-24.

Ramírez, M., Gouveia, E. y Lozada, J. (2011). El trabajo de campo estrategia metodológica para estudiar las comunidades. Omnia, 17(3), 9-22.

Ricoeur, P. (2000). La memoria, la historia, el olvido. México D. F.: Fondo de Cultura Económica.

Rodríguez, S. (2009). Producción, difusión y consolidación de la memoria oficial en Colombia. En A. Jiménez y F. Guerra (comps.), Las luchas por la memoria (pp. 95-126). Bogotá: Universidad Distrital Francisco José de Caldas; Centro de Memoria, Paz y Reconciliación Distrital.

Sánchez, O. y Rodríguez, S. (2009). Narrativa, memoria y enseñanza del conflicto armado colombiano: propuestas para superar las políticas del olvido y la impunidad. En D. Gómez y A. Serna (comps.), El papel de la memoria en los laberintos de la justicia, la verdad y la reparación (pp. 203-230). Bogotá: Universidad Distrital Francisco José de Caldas. 
Silva, O., Aponte, J., Cano, P., Ballesteros, L., Díaz, D., Escobar, C., González, G., Martínez, N., Pineda, A. y Pinto, J. (2010). Entre la memoria oficial y otras memorias: disputa de saber-poder en la enseñanza de las ciencias sociales. La primera violencia en Colombia. En C. Jilmar, J. Amador, I. Delgadillo y O. Silva, Emergencias de la memoria. Dos estudios sobre la infancia la escuela y la violencia (pp. 141-168). Bogotá: Universidad Distrital Francisco José de Caldas.

Todorov, T. (1995). Los abusos de la memoria. París: Paidós.

Uprimny, R. y Saffon, M. (2009). Reparaciones transformadoras, justicia distributiva y profundización democrática. En C. Díaz, N. Sánchez y R. Uprimny (eds.), Reparar en Colombia: los dilemas en contextos de conflicto, pobreza y exclusión (pp. 31-70). Bogotá: Centro Internacional para la Justicia Transicional (IстJ) y Centro de Estudios de Derecho, Justicia y Sociedad.

Zuluaga, O. y Marín, D. (2006). Memoria colectiva: memoria activa del saber pedagógico. Educación y Ciudad, 10, 63-86. 